\title{
People with Spinal Cord Injury in the Netherlands
}

\author{
Marcel W.M. Post, PhD, Carla F. Nooijen, PhD, Karin Postma, PT, PhD, Jos Dekkers, Frans Penninx, \\ Rita J.G. van den Berg-Emons, PhD, and Henk J. Stam, MD, PhD
}

\section{EPIDEMIOLOGY OF SPINAL CORD INJURY IN THE NETHERLANDS}

The estimated incidence of traumatic spinal cord injury (SCI) was 14.0 per million per year in the Netherlands in 2010, or 11.7 per million per year for those surviving the acute phase. ${ }^{1}$ The incidence of traumatic SCI seems rather stable as an earlier study reported an estimated incidence of 10.4 per million per year surviving the acute phase in $1994{ }^{2}$ The incidence of nontraumatic SCI in the Netherlands is unknown. However, a study on characteristics of persons with SCI admitted to an SCI department for initial inpatient rehabilitation found $54.7 \%$ of persons with SCI to have a nontraumatic lesion. ${ }^{3}$ Therefore, we assume that the incidence of nontraumatic SCI is slightly higher than the incidence of traumatic SCI. There are no reliable figures on the prevalence of SCI in the Netherlands.

Most cases of traumatic SCI admitted to acute care hospitals were falls (53\%) in 2010, followed by road traffic accidents $(22 \%)$ and sports $(14 \%){ }^{1}$ Within this last category, diving (27\%) and bicycling (23\%) were most common. Violence applied to only $2 \%$ of cases. Reported etiologies for nontraumatic SCI admitted to specialized rehabilitation centers were vascular diseases (28\%), spinal degeneration (26\%), inflammation $(17 \%)$, malignant tumor (17\%), and benign tumor $(11 \%){ }^{3}$

The most notable change between 1994 and 2010 was a strong increase in age at injury. The proportion of newly injured people older than 60 years was 30\% in 1994 compared with $52 \%$ in $2010 .{ }^{1,2}$ This increase in age at injury was associated with a growing proportion of falls as a cause of SCI and of more people with incomplete and cervical SCI. The decreasing proportion of traumatic SCI due to traffic incidents from $31 \%$ in 1994 to $22 \%$ in 2010 mirrors a decline in death and wounded people in traffic accidents in recent years in the Netherlands. ${ }^{4}$

The mortality rate during acute hospitalization was estimated to be $16 \%$ in $2010 .{ }^{1}$ In a cohort study of persons with SCI admitted to a specialized rehabilitation center who were expected to remain wheelchair dependent, the cumulative

From the Center of Excellence for Rehabilitation Medicine, the Brain Center Rudolf Magnus, University Medical Center Utrecht and De Hoogstraat Rehabilitation, Utrecht (MWMP); University of Groningen, University Medical Center Groningen, Center for Rehabilitation, Groningen (MWMP); Department of Rehabilitation Medicine, Erasmus University Medical Center Rotterdam (CFN, KP, RJGB-E, HJS) and Rijndam Rehabilitation Center (KP), Rotterdam; and Spinal Cord Injury Association of the Netherlands (Dwarslaesie Organisatie Nederland), Groningen (JD, FP), the Netherlands.

All correspondence and requests for reprints should be addressed to: Marcel W.M. Post, PhD, De Hoogstraat Rehabilitation, Rembrandtkade 10, 3583TM Utrecht, the Netherlands.

Financial disclosure statements have been obtained, and no conflicts of interest have been reported by the authors or by any individuals in control of the content of this article.

Copyright (C) 2017 Wolters Kluwer Health, Inc. All rights reserved.

ISSN: 0894-9115

DOI: 10.1097/PHM.0000000000000619 mortality was $12 \%$ at 5 years after injury. ${ }^{5}$ Main causes of death were cardiovascular disease (37\%), pulmonary disease $(30 \%)$, and neoplasm $(15 \%){ }^{5}$

\section{THE PATIENT JOURNEY THROUGH THE CHAIN OF CARE}

The typical journey through the health care system in the Netherlands of an adult with thoracic SCI due to a traffic accident (let us call her "Jane") begins with the injury. We may assume that Jane has the compulsory basic health insurance, which covers the costs (over the self-contribution level of 385 Euros a year) of trauma care and acute hospital care, rehabilitation and follow-up, most medical devices, and primary care (physician and registered nurse). Within 15 minutes after receiving the alarm, the emergency service should have arrived to provide Jane with first aid. In most cases, the emergency service will arrive by ambulance, but dependent on the location and the nature of the accident, it can also arrive by helicopter. The ambulance will convey Jane to the emergency department of a nearby hospital or trauma center. At this department, it is decided if and when Jane is referred to the intensive care.

A total of 94 intensive care units are spread over the country, including 8 university hospitals and 3 other level I trauma centers. Because Jane shows symptoms of spinal cord damage, she will be transported to one of these 11 level I trauma centers. She will probably receive stabilizing spinal surgery. Dependent on local arrangements, spinal surgery will be performed by orthopedic surgeons, trauma surgeons, or neurosurgeons, and Jane will stay at the respective ward afterwards. Median stay in the acute care hospital for patients with TSCI was 17 days (2010). ${ }^{1}$

Once medically stable, Jane will likely be transferred to 1 of the 8 rehabilitation centers with a specialization in SCI rehabilitation. These centers are spread over the country, so that she will probably stay in a center within a 1-hour drive by car from home. Post-acute SCI rehabilitation in these centers is comprehensive and multidisciplinary, consisting of medical, functional, and psychosocial rehabilitation. All relevant disciplines are available, for example, physical therapy, occupational therapy, recreation and leisure therapy, sports therapy, psychology, and social work. Length of stay in the rehabilitation center is strongly dependent on the type of SCI, but will be approximately 4 to 6 months for someone with complete paraplegia and up to 6 to 9 months for someone with complete tetraplegia.

After discharge from inpatient rehabilitation, Jane will receive outpatient rehabilitation for a further few months. If applicable, advice concerning return to work, advice for the provision of a wheelchair, and domestic adaptations will be part of inpatient or outpatient rehabilitation. 
Reintegration of persons with disabilities into the workforce is a priority of the Dutch government, and a variety of community services are available to support this process. ${ }^{6}$ There is, however, no reintegration service specific for persons with SCI. If Jane needs basic medical care after completion of initial inpatient and outpatient rehabilitation, such as in the case of an uncomplicated urinary tract infection, this is taken care of by a general practitioner (primary care physician, family physician). Rehabilitation aftercare is available, and Jane will probably return to the rehabilitation center for follow-up consultation 1 or 2 times in the first year. After the first year, the frequency of follow-up is agreed upon by patient and doctor. Some rehabilitation centers are implementing a system of standard structured multidisciplinary follow-up. If distance between home and center is an issue, Jane may be referred for follow-up to a nonspecialized rehabilitation center.

\section{LIVING WITH SCI}

In general, people with SCI will return to school if they are of school age. Accessibility problems may occur, in particular in case of older buildings, but in general, buildings and classrooms are more or less wheelchair accessible. Parents can apply for a budget to allow for some assistance for their child during school time. In exceptional cases, say if a child requires much assistance or has cognitive disabilities too, children with SCI are eligible for placement in special schools for children with severe physical disabilities. ${ }^{\text {? }}$

Return to work after SCI is far from complete in the Netherlands. A recent survey among members of the patient's organization showed that $72 \%$ of those employed at the time of their SCI returned to work for some time after their SCI, and $51 \%$ of the participants of working age had paid work for more than $1 \mathrm{~h} / \mathrm{wk}$ at the time of the survey. Of them, $27 \%$ worked full time ( $>35 \mathrm{~h} / \mathrm{wk})$, which is markedly less compared with $79 \%$ of those having paid work working fulltime before SCI. ${ }^{8}$ The general Dutch unemployment rate of persons with disabilities is among the Organisation for Economic Co-operation and Development average, but the poverty rate in this group at $12 \%$ is relatively low. ${ }^{9}$

There are no particular hindrances for persons with SCI to get married and have children. In the same survey, the participants' current civil status was married $(58 \%)$ or in a registered partnership (9\%), single $(20 \%)$, divorced $(5 \%)$, and widowed/ widower $(8 \%){ }^{8}$ The proportion of married persons with SCI is higher than that in the general population 20 to 64 years of age $(50 \%) .{ }^{10}$ Many persons with SCI have children, born before or after the SCI. Fertility treatment is covered by the basic health insurance.

From the same survey, we know that $48 \%$ of the participants performed unpaid (volunteer) work, and 13\% were in education. Further 58\% participated in sports, $84 \%$ performed outdoor leisure activities, $69 \%$ visited friends or family, $73 \%$ received visits from friends or family, and $93 \%$ had contacts by telephone or computer, all at least once a week. ${ }^{8}$

\section{THE HEALTH AND REHABILITATION SYSTEM}

Overall, the health care system in the Netherlands is well organized and of good quality. Health insurance is managed by private companies (usually nonprofit). Basic health insurance is compulsory and reaches $99 \%$ of the population. The fee is approximately 100 Euros a month, and there is a compulsory self-contribution of minimum 385 Eros and maximum 500 Euros (primary care consultations are exempted from this deductible). Slightly less than $90 \%$ has some form of supplementary health insurance, for example, to cover physical therapy, dental care, some medical devices, or complimentary medicine. Residential care, for example, nursing homes and long-stay psychiatry, is covered through taxation. Under certain conditions, it is possible for people in need of help with activities of daily living (showering, dressing, etc.) or other nursing care to apply for money to hire one's own help instead of care delivered by a home care agency. People who can handle the complexities of the system are thus able to organize their own maximally person-centered and flexible care. ${ }^{11}$ Provision of wheelchairs and other transportation modalities and domestic adaptations is the responsibility of the local municipalities. There is 1 primary care physician per 2379 inhabitants and, including all specialties, 3 physicians per 1000 inhabitants. $^{12,13}$

\section{WHAT IS THE STATE OF SPECIALIZED CARE?}

In general, the specialized care for persons with SCI is sufficient, and necessary treatments and devices are available. Peer counseling is a recent development, and now half of the SCI centers have a paid peer counselor. A challenge the rehabilitation system is facing at the moment is the growing number of older persons with SCI. Elderly are more often than younger persons discharged to nursing homes for rehabilitation, which do not have the specialized care for persons with SCI. ${ }^{1}$ It is, however, unclear whether treatment of the elderly with SCI is insufficient.

The economic crisis has led to budget cuts, and as a result, reimbursements of some services have become more limited, such as physical therapy in the community and sports wheelchairs. This has led to an increase of out-of-pocket costs. From January 2015, home care services have become the responsibility of local governments, together with a major budget cut, and the consequences of this change have to be awaited. Overall, it is a challenge to keep the services for people with disabilities from further deterioration.

\section{THE SOCIAL RESPONSE TO SCI}

The Netherlands have a complex social security system of the sort commonly found in high-resource European countries. $^{14}$ Government buildings and (partly) publicly financed buildings, such as museums, theaters, and hospitals, are mostly well accessible. Other public buildings (restaurants, pubs, hotels, shops) are, however, poorly accessible in many cases. With the exception of the subways in the big cities, the public transport system is poorly accessible. Streets in the older cities and private buildings are often difficult to access by wheelchair.

Figures on social attitudes toward persons with SCI are lacking. Although discrimination of people with disabilities is prohibited by the law, they often have difficulty finding a job. Research into discrimination of persons with SCI in the Netherlands is sparse. The only data are the aforementioned survey in which participants were asked the question: "People with disabilities such as mine are discriminated against 
in this country," on which question $18 \%$ provided an affirmative response. It is not possible to put this into perspective as data from other countries are lacking.

\section{THE INTERNATIONAL SPINAL CORD INJURY (InSCI) COMMUNITY SURVEY}

\section{What Do We Hope to Gain from Participating in the InSCI Study?}

The topics of International Spinal Cord Injury (InSCI) are generally well researched in the Netherlands. ${ }^{8,15-17}$ The added value of participation in InSCI is in (1) the opportunity to compare the situation of persons with SCI in the Netherlands with that in other countries and (2) the options of more in-depth multinational analyses of add-on topics. Three rehabilitation centers with a specialization in SCI rehabilitation, Rijndam Rehabilitation in Rotterdam, De Hoogstraat Rehabilitation in Utrecht, and the Center for Rehabilitation of the University Medical Center Groningen, will participate in this study. With these 3 centers in the Western, Middle, and Northern part of the Netherlands, respectively, the combined catchment area covers a large and representative part of the country, including both urban and rural areas.

A database including all former patients of these centers will be compiled, and the survival status of these persons will be checked. The inclusion and exclusion criteria are described in the general InSCI protocol paper in this issue. ${ }^{18}$ From this database, a random sample of 500 survivors will be drawn, so that with an expected response rate of $50 \%$, the target sample size of 250 will likely be reached. If necessary, an additional sample will be drawn.

Selected persons will be invited by postal mail. They will be provided with a link to a protected local Web site to complete the questionnaire online, or they can complete a pencil-and-paper version of the questionnaire and return it using a prepaid envelope. Unpublished data from a recent study showed that $98 \%$ of all people with long-standing SCI between 18 and 65 years of age have a computer and Internet access, ${ }^{15}$ but in another recent study in which participants were offered both response options, half chose the paper-and-pencil version. ${ }^{8}$ A maximum of 2 reminders will be sent. To enhance participation, the survey will be announced in various relevant magazines.

\section{The National Study Protocol}

Two options for a national module are under discussion. Dependent on the willingness of other InSCI countries and groups to join 1 or more of these topics, a final choice will be made.

(1) Physical activity. In an earlier study in the Netherlands, (the course in) physical activity level has been assessed with an accelerometry-based activity monitor in a rather small group of wheelchair-dependent people with SCI (40 persons). ${ }^{19}$ In this add-on, we want to explore physical activity levels in a larger representative Dutch sample (with questionnaires and activity monitors) and in persons with SCI in other countries. This study will provide information on determinants of physical activity and on differences in physical activity level between countries.
(2) Participation. Following up on previous research on participation $^{20,21}$ and the previously mentioned finding that some people with SCI feel discriminated by society, we want to explore how perspectives on social integration vary between countries. Do persons with SCI feel stigmatized? What do they feel that persons with SCI should do themselves to reintegrate in society? How does this impact their quality of life? To answer these questions, a brief questionnaire on experienced stigma and attitudes toward society will be administered.

\section{CONCLUSION}

From the description given in this article, it should be clear that the health and social needs of persons with SCI appear to be well addressed. However, as also described previously, there remain, or have emerged in recent years, several challenges that have to be confronted. We hope participation in InSCI will put these themes on the agenda of policy makers.

\section{REFERENCES}

1. Nijendijk JH, Post MW, van Asbeck FW: Epidemiology of traumatic spinal cord injuries in the Netherlands in 2010. Spinal Cord 2014;52:258-63

2. van Asbeck FW, Post MW, Pangalila RF: An epidemiological description of spinal cord injuries in the Netherlands in 1994. Spinal Cord 2000;38:420-4

3. Osterthun R, Post MW, van Asbeck FW: Characteristics, length of stay and functional outcome of patients with spinal cord injury in Dutch and Flemish rehabilitation centres. Spinal Cord 2009; 47:339-44

4. Institute for road safety research. Available at: http://swov.nl. Accessed February 24, 2016

5. Osterthun R, Post MW, van Asbeck FW, et al: Causes of death following spinal cord injury during inpatient rehabilitation and the first five years after discharge. A Dutch cohort study. Spinal Cord 2014;52:483-8

6. Work and income. Available at: www.werk-en-inkomen.com/arbeidsongeschiktheid-uitleg. Accessed February 24, 2016

7. Barf HA, Verhoef M, Post MW, et al: Educational career and predictors of type of education in young adults with spina bifida. Int $J$ Rehabil Res 2004;27:45-52

8. Post M, Heunik M: Arbeidsparticipatie en arbeidssatisfactie van mensen met een dwarslaesie in Nederland in 2012, Utrecht, the Netherlands: De Hoogstaat Rehabilitation; 2013

9. OECD. Sickness, disability and work: breaking the barriers.

10. Statistics Netherlands. Available at: http://statline.cbs.nl/statweb/publication/? $\mathrm{vw}=\mathrm{t} \& \mathrm{dm}=\mathrm{s} \ln 1 \& \mathrm{pa}=37975 \& \mathrm{~d} 1=0-5,10,15,19 \& \mathrm{~d} 2=0 \& \mathrm{~d} 3=0,5,10,15,(1-4)-1 \& \mathrm{hd}=151214-$ $1158 \& \mathrm{hdr}=\mathrm{t} \& \mathrm{stb}=\mathrm{g} 1, \mathrm{~g} 2$. Accessed February 24,2016

11. White C: The personal Touch: The Dutch experience of personal health budgets, London: The Health Foundation; 2011

12. OECD Health statistics. Health at a Glance 2013: OECD Indicators. OECD Publishing; 2013 Available at: http://dx.doi.org/10.1787/health_glance-2013-en. Accessed February 24, 2016

13. Daley C, Gubb J: Healthcare systems: the Netherlands. Civitas. 2013. Available at: www.digitalezorg.n1/digitale/uploads/2015/03/netherlands.pdf. Accessed February 24, 2016

14. US Social Security Administration Office of Retirement and Disability Policy. Social Security Programs Throughout the World: Europe. Washington, DC: SSA; Available at: www.ssa.gov/policy/docs/progdesc/ssptw/2012-2013/europe/netherlands.html. Accessed February 24, 2016. Uaa, ed. 2012:214-218

15. Adriaansen JJ, van Asbeck FW, Lindeman E, et al: Secondary health conditions in persons with a spinal cord injury for at least 10 years: design of a comprehensive long-term cross-sectional study. Disabil Rehabil 2013;35:1104-10

16. van Leeuwen CM, Post MW, Westers P, et al: Relationships between activities, participation, personal factors, mental health, and life satisfaction in persons with spinal cord injury. Arch Phys Med Rehabil 2012;93:82-9

17. van Koppenhagen CF, Post MW, van der Woude LH, et al: Changes and determinants of life satisfaction after spinal cord injury: a cohort study in the Netherlands. Arch Phys Med Rehabil 2008;89:1733-40

18. Gross-Hemmi MH, Post MWM, Ehrmann C, et al: Study protocol of the International Spinal Cord Injury (InSCI) community survey. Am J Phys Med Rehabil 2017;96 (Suppl):S23-34

19. van den Berg-Emons RJ, Bussmann JB, Haisma JA, et al: A prospective study on physical activity levels after spinal cord injury during inpatient rehabilitation and the year after discharge. Arch Phys Med Rehabil 2008;89:2094-101

20. van de Ven L, Post M, de Witte L, et al: It takes two to tango: the integration of people with disabilities into society. Disabil Soc 2005;20:311-29

21. van de Ven L, Post M, de Witte L, et al: Strategies for autonomy used by people with cervical spinal cord injury: a qualitative study. Disabil Rehabil 2008;30:249-60 